\title{
Dietary changes and dietary supplement use, and underlying motives for these habits reported by colorectal cancer survivors of the Patient Reported Outcomes Following Initial Treatment and Long-Term Evaluation of Survivorship (PROFILES) registry
}

\author{
Martijn J. Bours ${ }^{1 *}$, Sandra Beijer ${ }^{2}$, Renate M. Winkels ${ }^{3}$, Fränzel J. van Duijnhoven ${ }^{3}$, Floortje Mols ${ }^{2,4}$, \\ José J. Breedveld-Peters ${ }^{1}$, Ellen Kampman ${ }^{3,5,6}$, Matty P. Weijenberg ${ }^{1}$ and \\ Lonneke V. van de Poll-Franse ${ }^{2,4}$ \\ ${ }^{1}$ Department of Epidemiology, GROW - School for Oncology and Developmental Biology, Maastricht University, \\ P.O. Box 616, 6200 MD, Maastricht, The Netherlands \\ ${ }^{2}$ Comprehensive Cancer Center the Netherlands, Netherlands Cancer Registry, Eindhoven, The Netherlands \\ ${ }^{3}$ Division of Human Nutrition, Wageningen University, Wageningen, The Netherlands \\ ${ }^{4}$ Department of Medical and Clinical Psychology, Tilburg University, Tilburg, The Netherlands \\ ${ }^{5}$ Department of Health Sciences, VU University Amsterdam, Amsterdam, The Netherlands \\ ${ }^{6}$ Department for Health Evidence, Radboud University Medical Center, Nijmegen, The Netherlands
}

(Submitted 11 November 2014 - Final revision received 4 March 2015 - Accepted 27 April 2015 - First published online 16 June 2015)

\section{Abstract}

In the present study, we aimed to describe dietary changes made post-diagnosis and current dietary supplement use by survivors of colorectal cancer (CRC), and explore the underlying motives for these lifestyle habits. Cross-sectional analyses were performed for 1458 stage I-IV CRC survivors of the Patient Reported Outcomes Following Initial Treatment and Long-Term Evaluation of Survivorship (PROFILES) registry, diagnosed between 2000 and 2009. Lifestyle, sociodemographic and clinical information was collected. Prevalence of and motivations for dietary changes and supplement use were assessed. Associations between lifestyle, sociodemographic and clinical variables were analysed by multivariable logistic regression. CRC survivors (57\% male) were on average 70 (SD 9) years of age and diagnosed 7 (SD 3 ) years ago. Dietary changes post-diagnosis were reported by $36 \%$ of the survivors and current supplement use by $32 \%$. Motivations for dietary changes were mostly cancer-related ( $44 \%$ reported 'prevention of cancer recurrence' as the main reason), while motivations for supplement use were less frequently related to the cancer experience (38\% reported 'to improve health and prevent disease in general' as the main reason). Dietary changes were significantly associated with dietary supplement use (OR $1 \cdot 5,95 \%$ CI 1·1, 2·1). Survivors who had received dietary advice, were non-smokers, under 65 years of age, and had no stoma were more likely to have changed their diet. Survivors who were female, had multiple co-morbidities, and no overweight or obesity were more likely to use supplements. In conclusion, many CRC survivors alter their diet post-diagnosis and use dietary supplements, in part for different reasons. Insights into motivations behind these lifestyle habits and characteristics of CRC survivors adopting these habits can improve the tailoring of lifestyle counselling strategies.

\section{Key words: Colorectal cancer survivors: Dietary habits: Dietary supplements: Lifestyle behaviours}

Colorectal cancer (CRC) is a common cancer ${ }^{(1)}$. It is the third most commonly diagnosed cancer worldwide ${ }^{(2)}$. Besides population ageing, unhealthy dietary habits and other lifestyle-related risk factors (e.g. obesity) are thought to contribute to the rising incidence of $\mathrm{CRC}^{(3,4)}$. Furthermore, due to screening practices and improved curative treatment modalities, CRC survival rates have also increased over the past decades ${ }^{(5)}$. The growing number of CRC survivors constitutes an important target population for health promotion efforts, since many CRC survivors experience persisting physical and psychosocial problems after diagnosis and treatment, which negatively affect their health status and quality of life ${ }^{(6-9)}$. Additionally, compared

Abbreviations: CRC, colorectal cancer; ECR, Eindhoven Cancer Registry; PROFILES, Patient Reported Outcomes Following Initial Treatment and Long-Term Evaluation of Survivorship; SES, socio-economic status.

*Corresponding author: Dr M. J. Bours, fax +31433884128, email m.bours@maastrichtuniversity.nl 
with individuals without a history of cancer, cancer survivors have an increased risk for developing new primary tumours and chronic diseases, which may partly be attributed to an unhealthy lifestyle and body composition ${ }^{(10,11)}$. Therefore, promoting the adoption of a healthy lifestyle after a cancer diagnosis has the potential to favourably influence prognosis, co-morbidity and overall well-being $^{(3,12)}$

A cancer diagnosis has been referred to as a 'teachable moment' for motivating individuals to make behavioural changes for improving their health and well-being ${ }^{(11)}$. Research has shown that CRC survivors express the need for and actively seek advice and guidance on healthy lifestyle behaviours, including dietary requirements, in the period shortly after the end of initial treatment ${ }^{(13)}$. Nutrition and physical activity guidelines for cancer survivors are available ${ }^{(14-16)}$, which are intended to help health professionals counsel survivors about healthy lifestyle choices. Nevertheless, CRC survivors report confusion regarding the content and relevance of received advice about their personal lifestyle ${ }^{(13)}$. Directions for future research to improve existing guidelines have recently been suggested to enable better individual tailoring of lifestyle $\operatorname{advice}^{(17)}$.

A necessary first step towards improved tailoring of lifestyle advice for CRC survivors is characterisation of their dietary habits and other important lifestyle behaviours. Moreover, changes in lifestyle behaviours after diagnosis, motivations behind these changes, and interrelationships between these behaviours need to be characterised. The latter is of particular interest since cancer survivors may be inclined to make a healthful change in more than one behaviour to increase their sense of control over their lives and reduce their perceived physical and mental vulnerability ${ }^{(18,19)}$.

The present population-based cross-sectional study aimed to describe lifestyle behaviours of CRC survivors, specifically focusing on dietary changes made post-diagnosis and current dietary supplement use. Motives for adopting these habits as well as associations of these habits with other lifestyle behaviours and clinical and sociodemographic factors were also explored.

\section{Methods}

\section{Setting and participants}

Data were collected within the Patient Reported Outcomes Following Initial Treatment and Long-Term Evaluation of Survivorship (PROFILES) registry for the physical and psychosocial impact of cancer and its treatment ${ }^{(20)}$. The PROFILES registry facilitates collection of longitudinal data in populationbased cohorts of different types of cancer survivors, including CRC (http://www.profilesregistry.nl). It is linked directly to the Eindhoven Cancer Registry (ECR), which routinely collects information from all newly diagnosed cancer patients in ten hospitals in the southern part of The Netherlands. The ECR is part of the nationwide Netherlands Cancer Registry. In December 2010, a prospective study in a cohort of CRC survivors was set up. All living stage I-IV CRC patients diagnosed in the ECR region between 2000 and 2009 were eligible for participation. Patients with known cognitive impairment or an unverifiable address were excluded. From 2010 onwards, yearly surveys were performed by means of self-administered questionnaires. The longitudinal study was conducted according to the guidelines laid down in the Declaration of Helsinki, and all procedures involving human subjects were approved by a certified local medical ethics committee. Before participation, written informed consent was obtained from all subjects, thereby agreeing to link their questionnaire data with clinical data about their cancer and cancer treatment history in the ECR.

In the present study, we present data from the third survey that was performed in December 2012 (Fig. 1). To this survey, specific items were added regarding dietary changes, use of dietary supplements, motives for dietary changes and supplement use, and advice received about diet and supplement use after CRC diagnosis. These items were not included in the first two surveys of the longitudinal study.

\section{Data collection}

Information letters or emails were sent in December 2012 to a total of 1774 CRC survivors to invite them to participate in the third survey of the ongoing longitudinal study (Fig. 1). Non-respondents were sent a reminder within 2 months. Respondents could choose between completing the survey either online via a Web-based PROFILES platform, for which they received a personal log-in code, or filling in a paper questionnaire and returning it by mail.

\section{Sociodemographic and clinical data}

Sociodemographic data included sex and date of birth, and information on current marital status, highest attained educational level, current occupation, and socio-economic status (SES). SES was categorised as low, medium or high based on individual fiscal data from the year 2000 on the economic value of the home and household income, aggregated per postal code ${ }^{(21)}$. An additional SES category was added for individuals who were institutionalised at the time of assessment. Clinical data related to the patient's history of CRC included date of diagnosis, tumour site (colon or rectum), tumour stage (I to IV) according to the tumour-node-metastasis classification, and primary treatments received (surgery, chemotherapy and/or radiotherapy). Additionally, co-morbidity at the time of the survey was collected via the Self-Administered Comorbidity Questionnaire ${ }^{(22)}$. Co-morbidity was classified into 0,1 , or 2 or more co-morbid conditions. The presence of a stoma at the time of the survey was assessed by an item from the CRC-specific module of the European Organization for Research and Treatment of Cancer Quality of Life Questionnaire (EORTC QLQ-CR38) $^{(23)}$.

\section{Dietary habits and other lifestyle behaviours}

Participants were asked to report whether they had made changes to their diet since their CRC diagnosis. If so, they could indicate whether they had increased or decreased intake of eleven food components (i.e. components such as 


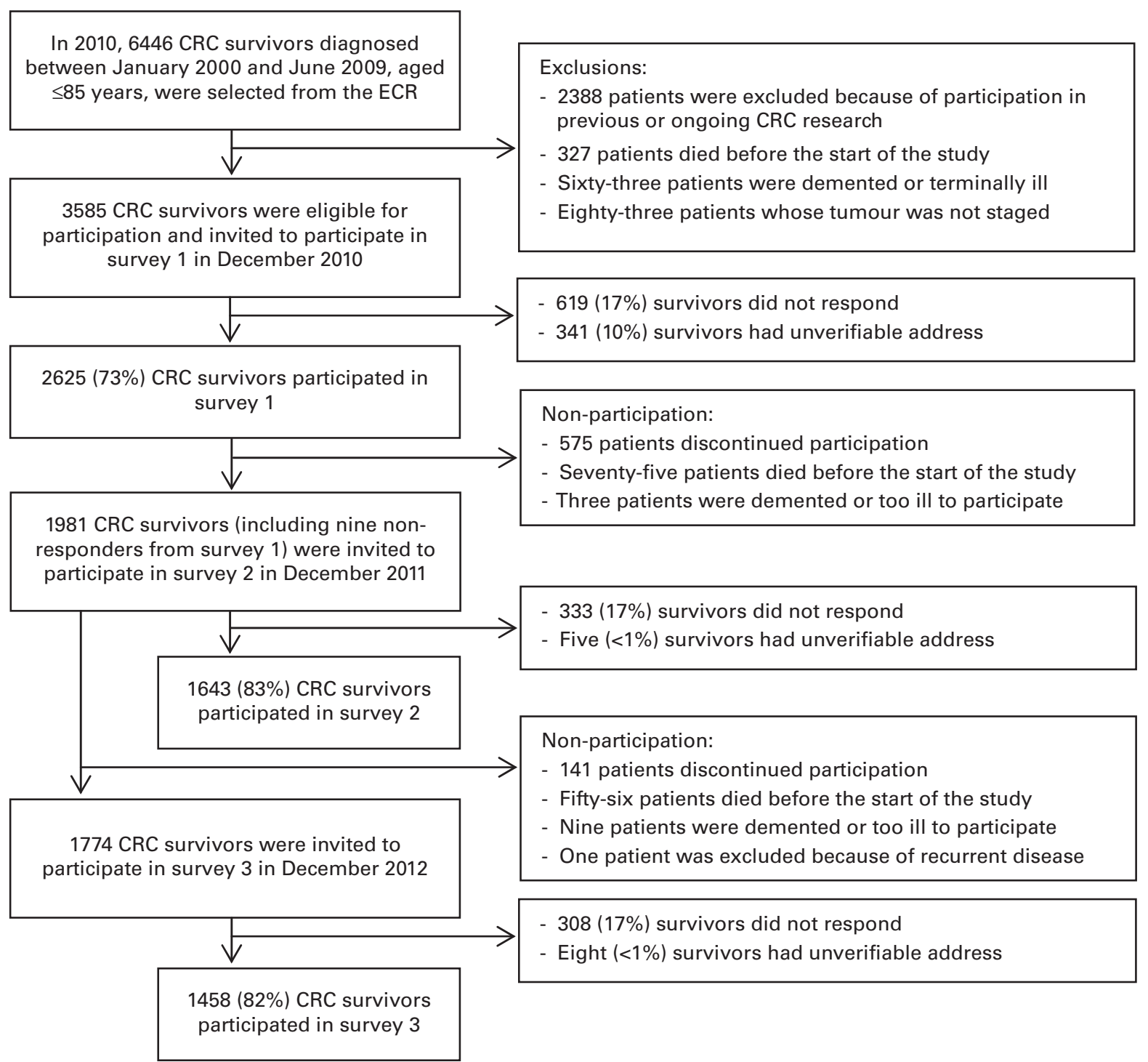

Fig. 1. Flow diagram of the study participants in the longitudinal study among colorectal cancer (CRC) survivors. For the present study, cross-sectional data from respondents to survey 3 are presented. ECR, Eindhoven Cancer Registry.

fat, fibres and wholegrains, and foods or food groups such as meat, fish, vegetables and fruit) and other nutritional products (i.e. milk and other dairy products, sugar, alcohol, water and salt). According to current dietary recommendations for cancer survivors ${ }^{(15,16)}$, self-reported dietary changes were classified as 'healthy changes' in case of less consumption of meat, fat, sugar, alcohol and salt, and more consumption of fruit, vegetables, fibres and wholegrain.

Additionally, participants were asked whether they used dietary supplements at the time of the survey and, if so, which supplement they were using (vitamin C, vitamin D, $\mathrm{Mg}$, fish oil, multivitamins/minerals, glucosamine and/or other) and since when they had started using it (i.e. since before or after their CRC diagnosis). Survivors who did not use dietary supplements were asked whether they had never done so, or whether they had used supplements in the past but stopped since before or after their CRC diagnosis.
The survey also included items about motivations for dietary changes and/or dietary supplement use. Survivors could indicate separately for dietary changes and supplement use one or more of the following motives: because of complaints related to the cancer; to prevent the cancer from recurring; to support therapy and recovery; or another reason (open-ended item). Survivors were also asked whether dietary advice was received after CRC diagnosis and whether a particular dietary regimen was being followed at the time of the survey, such as a vegetarian, vegan or organic diet, or a specific diet such as a low-fat, salt-free diet.

In addition, data on smoking, alcohol use and physical activity were collected. Current smoking and habitual alcohol use in the past 12 months were assessed. Physical activity was assessed using questions derived from the validated European Prospective Investigation into Cancer Physical Activity Questionnaire $^{(24)}$. Participants were asked to report how much 
time ( $\mathrm{h} /$ week) in the past year they had spent on average on walking, cycling, gardening, housekeeping and sports. To determine adherence to the Dutch Standard for Healthy Physical Activity, i.e. at least $150 \mathrm{~min}$ of moderate-to-vigorous physical activity per week, the duration of moderate-to-vigorous physical activity in min/week was calculated according to previously described methods ${ }^{(25)}$. The Dutch Standard is comparable to current physical activity guidelines for cancer survivors from the American College of Sports Medicine ${ }^{(14)}$, the American Cancer Society ${ }^{(15)}$, and the World Cancer Research Fund/ American Institute for Cancer Research (WCRF/AICR) ${ }^{(16)}$.

Furthermore, self-reported body height $(\mathrm{cm})$ and weight $(\mathrm{kg})$ were used to determine BMI $\left(\mathrm{kg} / \mathrm{m}^{2}\right)$ as a measure of body fatness. BMI was categorised as underweight (BMI $<18.5$ $\left.\mathrm{kg} / \mathrm{m}^{2}\right)$, normal weight $\left(18.5 \leq \mathrm{BMI}<25 \mathrm{~kg} / \mathrm{m}^{2}\right)$, overweight $\left(25 \leq \mathrm{BMI}<30 \mathrm{~kg} / \mathrm{m}^{2}\right)$ or obesity $\left(\mathrm{BMI} \geq 30 \mathrm{~kg} / \mathrm{m}^{2}\right)$.

\section{Statistical analyses}

Data on sociodemographic and clinical characteristics, dietary habits, and other lifestyle-related variables are reported as means and standard deviations, medians and interquartile ranges, or absolute $(n)$ and relative (\%) frequencies, depending on the measurement level. Descriptive statistics were calculated for the total study population, as well as subpopulations stratified according to dietary changes post-diagnosis and/or current dietary supplement use. To examine which factors were associated with dietary changes and supplement use, we performed multivariable logistic regression analyses. First, a binary logistic regression analysis was performed to assess whether dietary changes (yes/no) were associated with current dietary supplement use (yes/no), adjusted for other lifestyle variables and clinical and sociodemographic characteristics. Additionally, a multinomial logistic regression analysis was performed to assess whether changes classified as healthy dietary changes were associated with supplement use (dietary changes were categorised as no changes, unhealthy or less than seven healthy changes, and seven or more healthy changes).

Second, a multinomial logistic regression analysis was performed to assess whether a single nominal dependent variable with four mutually exclusive categories, based on combinations of dietary changes (yes/no) and current supplement use (yes/no), was associated with other lifestyle variables and clinical and sociodemographic characteristics as independent variables. In this analysis, subgroups of survivors who reported (1) dietary changes only, (2) supplement use only or (3) both dietary changes and supplement use were compared with the reference group of survivors who reported neither dietary changes nor dietary supplement use. Independent variables for the logistic regression analyses were selected based on previously reported data from a similar cohort of CRC survivors $^{(26)}$. Variables included in the regression models were current dietary regimen (yes/no), dietary advice received (yes/no), excess weight (yes/no, i.e. BMI $\geq 25$ or $<25 \mathrm{~kg} / \mathrm{m}^{2}$ ), adherence to the Dutch physical activity guideline (yes/no), current smoking (yes/no), current alcohol use (yes/no), age ( $<65$ or $\geq 65$ years), sex, SES (low/medium/high/institutionalised), co-morbidities $(0 / 1 / \geq 2)$, tumour stage (I to IV), tumour site (colon/rectum), time since diagnosis $(<5$ or $\geq 5$ years), chemotherapy (yes/no), and presence of a stoma (yes/no). For sufficient power $(>80 \%$ ), the minimum number of participants per independent variable in the logistic regression models was at least ten. Adjusted OR with corresponding 95\% CI are presented. Relevant assumptions for the logistic regression analyses were tested; none was shown to be violated. Additional multinomial regression analyses were performed stratified for years since diagnosis ( $<5$ years $v$. $\geq 5$ years).

All analyses were performed with IBM SPSS Statistics 20 for Windows. $P$ values $<0.05$ were considered as statistically significant.

\section{Results}

As shown in Fig. 1, of the total of 1774 eligible CRC survivors, 1458 participated in the survey ( $82 \%$ response rate). Questionnaires were returned either online ( $n$ 551, 38\%) or on paper (n 907, 62\%). Respondents were not meaningfully different $(<5 \%)$ from non-respondents with regard to age, sex, SES, time since CRC diagnosis, tumour stage, tumour site, and primary treatments received (data not shown).

\section{Participant characteristics}

\section{Sociodemographic and clinical characteristics}

Survivors were on average $70 \cdot 2$ (SD 9) years of age and $57 \%$ was male (Table 1). The largest proportion of CRC survivors was 65 years or older (72\%) and had a medium to high SES (19\% low SES, $40 \%$ medium SES and $40 \%$ high SES). The average time since CRC diagnosis was 6.9 (SD 3) years, and $60 \%$ of the respondents were at least 5 years from diagnosis. More survivors had been diagnosed with a tumour in the colon $(59 \%)$ than in the rectum (41\%). Most tumours were localised or only locally spread without distant metastases (31\% stage I, 36\% stage II, 30\% stage III and 3\% stage IV). Practically all survivors had undergone surgery (99.6\%), and $30 \%$ had also received chemotherapy and $33 \%$ radiotherapy. The presence of a stoma at the time of the survey was reported by $24 \%$ of the survivors, and the presence of at least one co-morbid condition was reported by $75 \%$.

\section{Dietary habits and other lifestyle behaviours}

In total, $36 \%$ of the CRC survivors reported having changed their diet since diagnosis, and $32 \%$ reported to use dietary supplements (Table 1). Of the survivors who did not use dietary supplements, $97 \%$ reported to have never used them and only $3 \%$ indicated to have stopped using supplements after their CRC diagnosis. More than half of the survivors (53\%) reported only dietary changes (21\%), only supplement use $(17 \%)$ or both (15\%). Survivors who solely reported dietary changes were more often male (61\%) than female (39\%), whereas survivors who reported both dietary changes and supplement use were more often female (61\%) than male (39\%). Adherence to a specific dietary regimen was reported by $12 \%$ of the survivors; this mostly concerned some form 
Table 1. Characteristics of the total study population of colorectal cancer (CRC) survivors and stratified according to the combinations of self-reported dietary changes since CRC diagnosis and current dietary supplement use

(Number of participants and percentages)

\begin{tabular}{|c|c|c|c|c|c|c|c|c|c|c|}
\hline & \multicolumn{2}{|c|}{ Total group } & \multicolumn{2}{|c|}{$\begin{array}{l}\text { Dietary } \\
\text { changes: no; } \\
\text { supplement } \\
\text { use: no }\end{array}$} & \multicolumn{2}{|c|}{$\begin{array}{l}\text { Dietary } \\
\text { changes: yes; } \\
\text { supplement } \\
\text { use: no }\end{array}$} & \multicolumn{2}{|c|}{$\begin{array}{c}\text { Dietary } \\
\text { changes: no; } \\
\text { supplement } \\
\text { use: yes }\end{array}$} & \multicolumn{2}{|c|}{$\begin{array}{c}\text { Dietary } \\
\text { changes: yes; } \\
\text { supplement } \\
\text { use: yes }\end{array}$} \\
\hline & $n$ & $\%$ & $n$ & $\%$ & $n$ & $\%$ & $n$ & $\%$ & $n$ & $\%$ \\
\hline Total & 1458 & 100 & 647 & 47 & 289 & 21 & 231 & 17 & 199 & 15 \\
\hline \multicolumn{11}{|l|}{ Sex } \\
\hline Male & 834 & 57 & 434 & 67 & 176 & 61 & 108 & 47 & 78 & 39 \\
\hline Female & 624 & 43 & 213 & 33 & 113 & 39 & 123 & 53 & 121 & 61 \\
\hline \multicolumn{11}{|l|}{ Age } \\
\hline$<65$ years & 402 & 28 & 174 & 27 & 97 & 34 & 60 & 26 & 62 & 31 \\
\hline$\geq 65$ years & 1055 & 72 & 472 & 73 & 192 & 66 & 171 & 74 & 137 & 69 \\
\hline \multicolumn{11}{|c|}{ Socio-economic status } \\
\hline Low & 263 & 19 & 114 & 18 & 55 & 19 & 38 & 17 & 32 & 17 \\
\hline Medium & 569 & 40 & 262 & 42 & 118 & 41 & 85 & 39 & 72 & 37 \\
\hline High & 561 & 40 & 239 & 38 & 110 & 38 & 95 & 43 & 86 & 44 \\
\hline Institutionalised & 25 & 2 & 11 & 2 & 4 & 1 & 3 & 1 & 4 & 2 \\
\hline \multicolumn{11}{|c|}{ Years since CRC diagnosis } \\
\hline$<5$ years & 576 & 40 & 272 & 42 & 110 & 38 & 85 & 37 & 72 & 36 \\
\hline$\geq 5$ years & 879 & 60 & 374 & 58 & 178 & 62 & 146 & 63 & 127 & 64 \\
\hline \multicolumn{11}{|l|}{ Tumour localisation } \\
\hline Colon & 865 & 59 & 385 & 59 & 159 & 55 & 148 & 64 & 113 & 57 \\
\hline Rectum & 593 & 41 & 262 & 41 & 130 & 45 & 83 & 36 & 86 & 43 \\
\hline \multicolumn{11}{|l|}{ Tumour stage } \\
\hline Stage I & 445 & 31 & 198 & 32 & 97 & 35 & 77 & 34 & 48 & 25 \\
\hline Stage II & 509 & 36 & 220 & 35 & 98 & 35 & 80 & 36 & 76 & 39 \\
\hline Stage III & 421 & 30 & 192 & 31 & 79 & 28 & 59 & 26 & 62 & 32 \\
\hline Stage IV & 41 & 3 & 17 & 3 & 6 & 2 & 9 & 4 & 7 & 4 \\
\hline \multicolumn{11}{|l|}{ Treatment } \\
\hline Surgery & 1452 & 100 & 643 & 99 & 289 & 100 & 231 & 100 & 199 & 100 \\
\hline Chemotherapy & 434 & 30 & 201 & 31 & 80 & 28 & 66 & 29 & 61 & 31 \\
\hline Radiotherapy & 476 & 33 & 208 & 32 & 107 & 37 & 67 & 29 & 68 & 34 \\
\hline \multicolumn{11}{|l|}{ Stoma } \\
\hline Yes & 295 & 24 & 141 & 25 & 48 & 19 & 52 & 25 & 42 & 26 \\
\hline No & 930 & 76 & 422 & 75 & 201 & 81 & 160 & 75 & 120 & 74 \\
\hline \multicolumn{11}{|l|}{ Co-morbidities } \\
\hline 0 & 351 & 25 & 186 & 30 & 71 & 25 & 45 & 21 & 40 & 21 \\
\hline 1 & 429 & 31 & 198 & 32 & 90 & 32 & 67 & 31 & 57 & 29 \\
\hline$\geq 2$ & 601 & 44 & 243 & 39 & 120 & 43 & 103 & 48 & 98 & 50 \\
\hline \multicolumn{11}{|c|}{ Adherence to the Dutch physical activity norm } \\
\hline Yes & 1222 & 91 & 558 & 92 & 242 & 90 & 200 & 92 & 173 & 92 \\
\hline \multicolumn{11}{|l|}{ Current smoking } \\
\hline Yes & 154 & 11 & 84 & 13 & 20 & 7 & 20 & 9 & 15 & 8 \\
\hline \multicolumn{11}{|l|}{ Current alcohol use } \\
\hline Yes & 1036 & 72 & 491 & 77 & 195 & 68 & 172 & 75 & 133 & 68 \\
\hline \multicolumn{11}{|l|}{ Excess weight } \\
\hline Yes & 930 & 65 & 450 & 70 & 191 & 67 & 123 & 54 & 116 & 59 \\
\hline \multicolumn{11}{|c|}{ Current dietary regimen } \\
\hline Yes & 162 & 12 & 25 & 4 & 58 & 21 & 21 & 9 & 50 & 27 \\
\hline \multicolumn{11}{|c|}{ Dietary advice received } \\
\hline Yes & 233 & 17 & 64 & 10 & 73 & 26 & 29 & 13 & 63 & 33 \\
\hline
\end{tabular}

of low-fat and/or low-salt diet rich in fruit, vegetables and fibres. Only $17 \%$ of the survivors reported to have received advice after their CRC diagnosis with regard to diet and dietary supplement use. Nearly all of the CRC survivors who reported having received dietary advice were satisfied with the advice received (96\%), and the majority of the survivors who did not receive advice reported to have had no need for such advice (84\%). Current smoking was reported by $11 \%$ of the survivors, habitual alcohol use in the past year by $72 \%$ of the survivors, and the majority of the survivors (91\%) adhered to the Dutch physical activity guideline according to selfreported physical activity levels (Table 1 ).
Excess weight (BMI $\geq 25 \mathrm{~kg} / \mathrm{m}^{2}$ ) was present in $65 \%$ of the CRC survivors, being more prevalent in men (71\%) than in women (54\%). In total, $0 \cdot 3 \%$ of the survivors were underweight $\left(\mathrm{BMI}<18.5 \mathrm{~kg} / \mathrm{m}^{2}\right), 35 \%$ had normal weight $(18.5 \leq \mathrm{BMI}$ $\left.<25 \mathrm{~kg} / \mathrm{m}^{2}\right), 47 \%$ were overweight $\left(25 \leq \mathrm{BMI}<30 \mathrm{~kg} / \mathrm{m}^{2}\right)$ and $17 \%$ were obese $\left(\mathrm{BMI} \geq 30 \mathrm{~kg} / \mathrm{m}^{2}\right)$.

\section{Self-reported dietary changes and dietary supplement use}

Among the CRC survivors who changed their diet since diagnosis, 53\% reported to have changed their intake (either more or less) of all the eleven food components and other 
Table 2. Frequency and nature of self-reported dietary changes since colorectal cancer (CRC) diagnosis among the total number of CRC survivors reporting dietary changes

(Number of participants and percentages)

\begin{tabular}{|c|c|c|c|c|c|c|c|}
\hline \multirow{3}{*}{$\begin{array}{l}\text { Specific food } \\
\text { components and other } \\
\text { nutritional products }\end{array}$} & \multirow{2}{*}{\multicolumn{2}{|c|}{$\begin{array}{l}\text { Total number } \\
\text { of survivors } \\
\text { reporting a } \\
\text { dietary change }\end{array}$}} & \multicolumn{4}{|c|}{$\begin{array}{l}\text { Dietary changes since CRC diagnosis, } \\
\text { i.e. more or less intake }\end{array}$} & \multirow{3}{*}{$\begin{array}{l}\text { Healthy changes } \\
\% \text { of total }\end{array}$} \\
\hline & & & \multicolumn{2}{|c|}{ More } & \multicolumn{2}{|c|}{ Less } & \\
\hline & $n$ & $\%$ & $n$ & $\%$ of total & $n$ & $\%$ of total & \\
\hline Fat $^{*}$ & 427 & 86 & 14 & 3 & 413 & 97 & 97 \\
\hline Meat ${ }^{*}$ & 401 & 81 & 23 & 6 & 376 & 94 & 94 \\
\hline Fish & 388 & 78 & 342 & 88 & 45 & 12 & $\mathrm{n} / \mathrm{a}$ \\
\hline Vegetables $\dagger$ & 403 & 81 & 383 & 95 & 20 & 5 & 95 \\
\hline Fruit† & 408 & 82 & 374 & 92 & 33 & 8 & 92 \\
\hline Milk and other dairy products & 362 & 73 & 198 & 55 & 161 & 45 & $\mathrm{n} / \mathrm{a}$ \\
\hline Fibres and wholegrain $\dagger$ & 415 & 84 & 379 & 91 & 36 & 9 & 91 \\
\hline Sugar* & 379 & 77 & 16 & 4 & 362 & 96 & 96 \\
\hline Alcohol $^{*}$ & 339 & 69 & 15 & 5 & 322 & 95 & 95 \\
\hline Water & 386 & 78 & 343 & 89 & 42 & 11 & $\mathrm{n} / \mathrm{a}$ \\
\hline Salt $^{*}$ & 386 & 78 & 11 & 3 & 374 & 97 & 97 \\
\hline
\end{tabular}

n/a, Not applicable.

${ }^{*}$ According to current healthy diet recommendations for cancer survivors ${ }^{(15,16)}$, less consumption of fat, meat, sugar, alcohol and salt was regarded as healthy dietary changes since diagnosis.

† According to current healthy diet recommendations for cancer survivors ${ }^{(15,16)}$, more consumption of fruit, vegetables, and fibres and wholegrain was regarded as healthy dietary changes since diagnosis.

nutritional products mentioned. Most survivors reported to have started using less fat, meat, sugar, alcohol, and salt since their CRC diagnosis, while using more fish, vegetables, fruit, fibres and wholegrain, and water (Table 2). The median number of dietary changes classified as healthy changes' was 7 (Table 2). Regarding dietary supplement use, $41 \%$ reported to have been using supplements since before their CRC diagnosis and 59\% reported to have started using supplements since after their diagnosis. The median number of different types of supplements reported was 2 (interquartile range 1-3). Among the current supplement users, $48 \%$ reported to use one dietary supplement, $25 \%$ reported to use two different types of supplements, 13\% used three different supplements, and $14 \%$ used four or more different supplements. As shown in Table 3, use of multivitamins and minerals was most frequently reported by the survivors (50\%), followed by vitamin C (37\%), vitamin D (34\%), Mg (22\%), fish oil (19\%) and glucosamine (18\%). Nearly one-third of the survivors (32\%) reported the use of other dietary supplements, among which vitamin B supplements were the most common. No large differences in specific dietary changes or supplements used were observed between men and women (data not shown).

The most frequent reason reported by CRC survivors for having changed their diet after diagnosis (Fig. 2(a)) was to prevent the cancer from recurring (44\%). Other motives for dietary changes since CRC diagnosis were to support therapy and recovery (20\%), cancer-related complaints (19\%), or other reasons (17\%), such as to promote bowel movement, lose weight or improve general health. Motives reported for using dietary supplements (Fig. 2(b)) rarely concerned cancer-related complaints ( $4 \%$ ), but were more frequently to prevent the cancer from recurring (25\%), to support therapy and recovery $(33 \%)$, or other reasons $(38 \%)$. The most frequently mentioned other reasons for supplement use were to improve general health and prevent disease.

Table 3. Frequency and nature of self-reported use of dietary supplements among the total number of colorectal cancer (CRC) survivors reporting supplement use

(Number of participants and percentages)

\begin{tabular}{|c|c|c|c|c|c|c|}
\hline \multirow{3}{*}{$\begin{array}{l}\text { Specific supplements } \\
\text { used }\end{array}$} & & & \multicolumn{4}{|c|}{$\begin{array}{l}\text { Supplement use since before } \\
\text { or after CRC diagnosis }\end{array}$} \\
\hline & \multicolumn{2}{|c|}{ Total } & \multicolumn{2}{|c|}{ Before } & \multicolumn{2}{|c|}{ After } \\
\hline & $n$ & $\%$ & $n$ & $\%$ of total & $n$ & $\%$ of total \\
\hline Vitamin C & 161 & 37 & 81 & 52 & 75 & 48 \\
\hline Vitamin D & 148 & 34 & 51 & 34 & 94 & 65 \\
\hline $\mathrm{Mg}$ & 97 & 22 & 31 & 33 & 64 & 67 \\
\hline Fish oil & 83 & 19 & 37 & 45 & 46 & 55 \\
\hline Multivitamins/minerals & 218 & 50 & 96 & 45 & 115 & 55 \\
\hline Glucosamine & 77 & 18 & 36 & 51 & 35 & 49 \\
\hline Other* & 140 & 32 & 48 & 35 & 90 & 65 \\
\hline
\end{tabular}

*Vitamin B supplements were the most frequently reported type of other dietary supplements. 
(a)

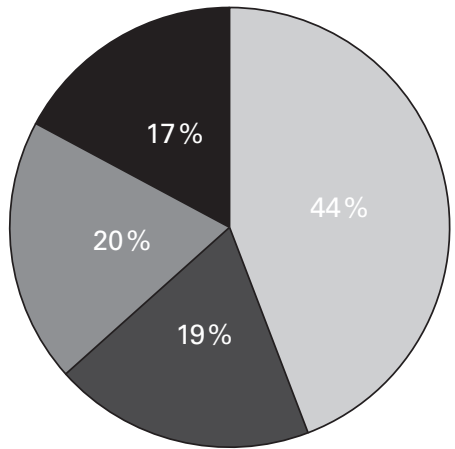

(b)

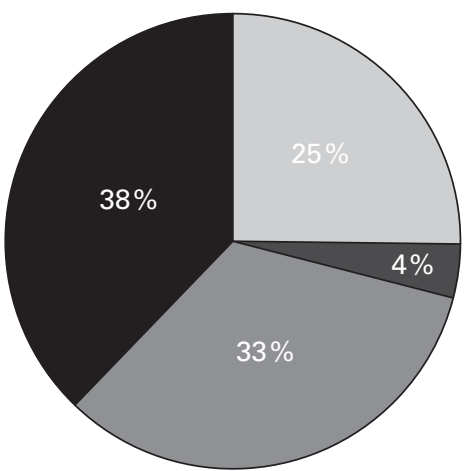

Fig. 2. Pie charts of motives self-reported by colorectal cancer survivors for having changed their diet post-diagnosis (a) or for currently using dietary supplements (b). $\square$, To prevent cancer recurrence; $\square$, complaints related to cancer; $\square$, to support therapy/recovery; $\square$, other.

Compared with all current supplement users, reasons reported for supplement use were similar in the subgroup of survivors who indicated to have started using supplements since after their diagnosis (data not shown).

\section{Associations between dietary changes, supplement use and other variables}

Self-reported dietary changes since CRC diagnosis were positively associated with current dietary supplement use (adjusted OR 1·5, 95\% CI 1·1, 2·1). Additionally, multinomial logistic regression analyses showed that self-reported supplement use was significantly more likely only among survivors who had reported seven or more healthy dietary changes since diagnosis (OR 1·8, 95\% CI 1·2, 2.6), compared with survivors who had reported no dietary changes (see online Supplementary Table S1). Furthermore, survivors who reported unhealthy or less than seven healthy dietary changes were significantly less likely to adhere to the Dutch physical activity norm (OR $0 \cdot 4,95 \%$ CI $0 \cdot 2,0 \cdot 8$ ) and to have a stoma (OR $0 \cdot 5,95 \%$ CI $0.3,0 \cdot 8$ ), and more likely to have had rectal cancer (OR 1·8, $95 \%$ CI 1·1, 2·8).

As shown in Table 4 , relative to the CRC survivors who reported neither dietary changes nor supplement use, survivors who only reported to have changed their diet since diagnosis were more likely to follow a dietary regimen (OR $7 \cdot 1,95 \%$ CI $3.9,12.9$ ) and to have received dietary advice (OR 3.6, $95 \%$ CI 2.3, 5.6), and less likely to be current smokers (OR 0.4, 95\% CI 0.2, 0.8), to be 65 years or older (OR $0 \cdot 7,95 \%$ CI $0 \cdot 5,1 \cdot 0)$ and to have a stoma (OR $0.5,95 \%$ CI 0.3 , $0 \cdot 8)$. When stratified for years since diagnosis, the significant association of dietary changes with smoking was only observed in survivors who were less than 5 years since diagnosis, whereas the significant associations with age and stoma were only observed in survivors who were 5 years or more since diagnosis (see online Supplementary Tables S2 and S3). Additionally, only in survivors who were less than 5 years since diagnosis (see online Supplementary Table S2), dietary changes were significantly less likely to be reported by the survivors adhering to the Dutch physical activity norm (OR 0.3, 95\% CI 0.1, 0.8).

Survivors who only reported dietary supplement use were more likely to follow a dietary regimen (OR $2 \cdot 5,95 \%$ CI $1 \cdot 2$, $5 \cdot 0$ ), to be female (OR $2 \cdot 5,95 \%$ CI $1.7,3.6$ ) and to have at least two co-morbidities (OR 1.6, 95\% CI 1·0, 2.6), and less likely to have excess weight (OR $0.5,95 \%$ CI $0.3,0.7$ ), compared with survivors who did not use supplements and had not changed their diet (Table 4). In the analyses stratified for years since diagnosis, the association of supplement use with female sex remained significant in all the strata; however, the significant association with excess weight was only significant in survivors who were less than 5 years since diagnosis (see online Supplementary Tables S2 and S3). Additionally, only in survivors who were less than 5 years since diagnosis (see online Supplementary Table S2), current supplement use was significantly less likely to be reported by survivors who had a higher tumour stage at diagnosis (OR 0.4, 95\% CI $0 \cdot 2,0.9$ for stage II and OR $0 \cdot 4,95 \%$ CI $0 \cdot 1,1 \cdot 0$ for stage III) and a rectal tumour (OR 0.4, 95\% CI 0.2, 0.9).

Finally, survivors who reported to have changed their diet as well as to use dietary supplements were more likely to follow a dietary regimen (OR $8 \cdot 8,95 \%$ CI 4.6, 16.8), to have received advice about diet and dietary supplements (OR 4.4, $95 \%$ CI $2 \cdot 7,7 \cdot 2$ ), and to be female (OR $2 \cdot 2,95 \%$ CI $1 \cdot 5,3 \cdot 4$ ), in comparison with survivors who reported neither dietary changes nor supplement use (Table 4). Similar results were found in the analyses stratified for years since diagnosis (see online Supplementary Tables S2 and S3), except for the finding that both self-reported dietary changes and supplement use were less likely in survivors with excess weight who were less than 5 years since diagnosis (OR 0.4, 95\% CI 0.2, 0.8; see online Supplementary Table S2).

Survivors who reported to have received dietary advice were significantly more likely to have made changes in their diet since diagnosis, both unhealthy or less than seven healthy changes (OR $3 \cdot 9,95 \%$ CI $2 \cdot 5,6 \cdot 1$ ) and seven or more healthy changes (OR $3 \cdot 1,95 \%$ CI $2 \cdot 1,4 \cdot 8$ ), as well as to have had rectal cancer and to have a stoma (OR 1.6, 95\% CI 1·1, 2.3 and OR $1.9,95 \%$ CI $1.3,3 \cdot 0$, respectively; see online Supplementary Table S4).

\section{Discussion}

In the present cross-sectional analysis, we observed that a sizeable proportion (53\%) of 1458 Dutch CRC survivors reported to have made dietary changes post-diagnosis or to 
Table 4. Associations of self-reported dietary changes since colorectal cancer (CRC) diagnosis and/or current dietary supplement use with other lifestyle-related, sociodemographic and clinical variables $(n 999) \dagger$

(Odds ratios and $95 \%$ confidence intervals)

\begin{tabular}{|c|c|c|c|c|c|c|}
\hline & \multicolumn{2}{|c|}{$\begin{array}{l}\text { Dietary changes: yes; } \\
\text { supplement use: no }\end{array}$} & \multicolumn{2}{|c|}{$\begin{array}{l}\text { Dietary changes: no; } \\
\text { supplement use: yes }\end{array}$} & \multicolumn{2}{|c|}{$\begin{array}{l}\text { Dietary changes: yes; } \\
\text { supplement use: yes }\end{array}$} \\
\hline & OR $\ddagger$ & $95 \% \mathrm{Cl}$ & OR $\ddagger$ & $95 \% \mathrm{Cl}$ & OR $\ddagger$ & $95 \% \mathrm{Cl}$ \\
\hline \multicolumn{7}{|l|}{ Lifestyle-related variables } \\
\hline \multicolumn{7}{|c|}{ Current dietary regimen (reference: no) } \\
\hline Yes & $7 \cdot 1^{*}$ & $3.9,12.9$ & $2 \cdot 5^{\star}$ & $1 \cdot 2,5 \cdot 0$ & $8 \cdot 8^{*}$ & $4 \cdot 6,16 \cdot 8$ \\
\hline \multicolumn{7}{|c|}{ Dietary advice received (reference: no) } \\
\hline Yes & $3.6^{*}$ & $2 \cdot 3,5 \cdot 6$ & 1.3 & $0.7,2 \cdot 2$ & $4 \cdot 4^{*}$ & $2 \cdot 7,7 \cdot 2$ \\
\hline \multicolumn{7}{|c|}{ Excess weight (reference: no) } \\
\hline Yes & 0.8 & $0.5,1.2$ & $0.5^{\star}$ & $0.3,0.7$ & 0.7 & $0.4,1 \cdot 1$ \\
\hline \multicolumn{7}{|c|}{ Adherence to the physical activity norm (reference: no) } \\
\hline Yes & 0.5 & $0.3,1.0$ & 0.9 & $0.5,1.8$ & 0.8 & $0.4,1.7$ \\
\hline \multicolumn{7}{|c|}{ Current smoking (reference: no) } \\
\hline Yes & $0 \cdot 4^{*}$ & $0.2,0.8$ & 0.6 & $0.3,1 \cdot 2$ & 0.7 & $0.4,1.5$ \\
\hline \multicolumn{7}{|c|}{ Current alcohol use (reference: no) } \\
\hline Yes & 0.8 & $0.5,1.2$ & 0.9 & $0.6,1.5$ & $1 \cdot 1$ & $0.7,1 \cdot 8$ \\
\hline \multicolumn{7}{|c|}{ Sociodemographic variables } \\
\hline \multicolumn{7}{|c|}{ Age (reference: $<65$ years) } \\
\hline$\geq 65$ years & $0.7^{\star}$ & $0.5,1.0$ & 0.9 & $0.6,1.4$ & 0.8 & $0.5,1.3$ \\
\hline \multicolumn{7}{|l|}{ Sex (reference: male) } \\
\hline Female & $1 \cdot 0$ & $0.7,1.5$ & $2 \cdot 5^{\star}$ & $1 \cdot 7,3.6$ & $2 \cdot 2^{*}$ & $1.5,3.4$ \\
\hline \multicolumn{7}{|c|}{ Socio-economic status (reference: low) } \\
\hline Medium & 0.9 & $0.6,1.5$ & 1.3 & $0.7,2 \cdot 2$ & $1 \cdot 1$ & $0 \cdot 6,2 \cdot 1$ \\
\hline High & 0.9 & $0.6,1.6$ & 1.5 & $0.9,2.6$ & 1.7 & $0.9,3.1$ \\
\hline Institutionalised & 0.7 & $0 \cdot 2,2 \cdot 7$ & 1.0 & $0.2,4.1$ & 0.9 & $0.2,4.5$ \\
\hline \multicolumn{7}{|l|}{ Clinical variables } \\
\hline \multicolumn{7}{|c|}{ Co-morbidities (reference: no co-morbidities) } \\
\hline 1 co-morbidity & $1 \cdot 2$ & $0.7,1 \cdot 8$ & 1.4 & $0.8,2 \cdot 2$ & $1 \cdot 2$ & $0.7,2 \cdot 2$ \\
\hline$\geq 2$ co-morbidities & 1.2 & $0.8,1.9$ & $1.6^{\star}$ & $1 \cdot 0,2 \cdot 6$ & $1 . \overline{5}$ & $0.9,2.6$ \\
\hline \multicolumn{7}{|l|}{ Stage (reference: I) } \\
\hline Stage II & $1 \cdot 1$ & $0.7,1 \cdot 7$ & 1.0 & $0.6,1.5$ & 1.4 & $0.8,2 \cdot 4$ \\
\hline Stage III & 1.3 & $0 \cdot 8,2 \cdot 3$ & 0.7 & $0.4,1.4$ & 1.7 & $0.9,3.3$ \\
\hline Stage IV & $1 \cdot 1$ & $0.4,3.4$ & 1.3 & $0.4,3.7$ & 1.5 & $0.4,5.4$ \\
\hline \multicolumn{7}{|c|}{ Localisation (reference: colon) } \\
\hline Rectum & 1.4 & $1 \cdot 0,2 \cdot 1$ & 0.8 & $0.5,1.3$ & $1 \cdot 2$ & $0.7,1.9$ \\
\hline \multicolumn{7}{|c|}{ Years since diagnosis (reference: $<5$ years) } \\
\hline$\geq 5$ years & 1.2 & $0 \cdot 8,1 \cdot 7$ & $1 \cdot 1$ & $0.8,1.6$ & 1.1 & $0.7,1 \cdot 7$ \\
\hline \multicolumn{7}{|c|}{ Chemotherapy (reference: no) } \\
\hline Yes & 0.9 & $0.5,1.4$ & 1.0 & $0.6,1 \cdot 8$ & 1.1 & $0.6,1.9$ \\
\hline \multicolumn{7}{|l|}{ Stoma (reference: no) } \\
\hline Yes & $0.5^{*}$ & $0.3,0.8$ & 1.0 & $0.6,1.6$ & 0.8 & $0.4,1.3$ \\
\hline
\end{tabular}

${ }^{*} P<0.05$.

† Model $\chi^{2}(\mathrm{df})=237.2(60), P<0.001 ; R^{2}$ (Cox and Snell): 0.211, $R^{2}$ (Nagelkerke): 0.230.

‡Reference category is no self-reported dietary changes since CRC diagnosis plus no self-reported current dietary supplement use.

currently use dietary supplements. CRC survivors who had changed their diet, especially those who mainly reported healthy changes, were more likely to also use dietary supplements. The majority of survivors reported changes in multiple food components or other nutritional products. Additionally, most survivors reported to use one or two dietary supplements, which typically were (multi-)vitamins and minerals. Interestingly, the reasons reported by survivors for having changed their diet or using dietary supplements appeared to be different. Dietary changes were most often reported to be related to prevention of cancer recurrence, whereas more general motivations, such as to improve health and prevent disease in general, were reported the most for dietary supplement use. Moreover, we observed that characteristics of CRC survivors who reported dietary changes and those who reported supplement use appeared to be partly dissimilar, and possibly also related to time since diagnosis. Self-reported dietary changes were significantly more likely among survivors who had received dietary advice, did not smoke, were younger than 65 years of age, and had no stoma. In contrast, current supplement use was significantly associated with being female, having no excess weight, and having at least two co-morbid conditions.

We observed that self-reported dietary changes were for the most part in line with current guidelines for healthy eating habits (i.e. limit consumption of energy-dense and salty foods, red and processed meat and alcohol, and eat more fruits, vegetables and wholegrain products) ${ }^{(15,16)}$. Based on our data, we could not distinguish between categories of certain foods (e.g. red and processed meat). Nevertheless, we believe that our findings can be interpreted as being in line with the dietary guidelines, as reporting of, for instance, 
less consumption of meat probably includes decreased consumption of red and processed meat, thus corresponding to the current guidelines. Studies on dietary habits in CRC survivors are relatively scarce. Our findings in a Dutch population of CRC survivors are largely comparable to the findings from previous observational studies in smaller samples or specific subgroups of CRC survivors performed in the USA, which showed that between 31 and $52 \%$ of CRC survivors reported to have changed their diet after diagnosis ${ }^{(27,28)}$, and between 28 and $49 \%$ reported to use supplements ${ }^{(27,29,30)}$. A noteworthy finding of the present study was that specific motivations reported by CRC survivors for dietary changes or for supplement use were found to differ. Our findings suggest that the reasons for changing the diet may be more cancer-related, while reasons for using supplements may be more general and not specifically related to the cancer experience. The psychological drivers that play a role in decision-making processes with regard to particular health behaviours is an understudied area of investigation in CRC survivors. Previously, Mullens et al. ${ }^{(27)}$ showed that changing diet and engaging in complementary therapies, which included taking dietary supplements (typically vitamins), were the two most important things that CRC survivors thought they could do to prevent recurrence and improve general health. This seems to agree with our findings, and indicates that it would be very relevant for health professionals to better understand the underlying reasons and intentions for adopting certain dietary habits when counselling CRC survivors about their diet and other lifestyle behaviours. More research in this area should be highly prioritised ${ }^{(31,32)}$.

Another interesting finding of the present study was that the CRC survivors who changed their diet appeared to differ in part from those who used dietary supplements. Previous studies on correlates of dietary habits among CRC survivors have mainly been performed in the USA. In a longitudinal study among 278 colon cancer survivors, it was found that older and female survivors were more likely to use supplements, and that increased fruit and vegetable intake was more likely among survivors who were older, unemployed and female ${ }^{(28,33)}$. Additionally, a population-based crosssectional study in 529 CRC survivors showed that female and better educated survivors treated with chemotherapy more often used supplements ${ }^{(29)}$. Furthermore, another crosssectional study in a population of mixed types of cancer survivors, including 116 CRC survivors, showed that dietary supplement use was more likely among female, older, nonsmoking and better educated survivors with more advanced disease receiving multiple cancer therapies ${ }^{(34,35)}$, and that dietary changes were more likely among younger and female survivors with a higher education ${ }^{(35)}$. The foregoing indicates that findings from previous, smaller studies are not straightforward and only partly consistent with our findings. For example, somewhat unexpectedly, we observed no significant association between education level and dietary changes and/ or supplement use. It seems to be difficult to specifically characterise CRC survivors who are receptive to adopt different dietary habits, as this probably depends on personal preferences, intentions, beliefs and cultural influences.
Moreover, we observed that a large proportion of CRC survivors $(83 \%)$ reported to have not received advice about diet and/or dietary supplement use after diagnosis, and also to have had no need for such advice. It is not unlikely that part of these survivors may wrongly believe that their dietary and other lifestyle habits are healthy. Therefore, an important task in practice would be to identify and better inform these individuals about a healthy diet and lifestyle and to motivate them into changing their lifestyle behaviour. Providing individual CRC survivors with appropriately tailored advice may be an important stimulus for inducing such behavioural changes, as we observed that dietary changes were more likely among survivors who had received dietary advice. Intriguingly, we observed that survivors with a stoma were more likely to report having received dietary advice, which might seem contradictory with the observation that survivors with a stoma were less likely to report dietary changes. However, this is probably a reflection of current practice, in which patients with a stoma receive the advice that changing their diet because of a stoma is not necessary.

The present study has several strengths and also limitations. The major strengths are its large sample size and the high response rate, which was due to the fact that our analyses were performed as part of an ongoing longitudinal study that collects data through the well-established PROFILES registry ${ }^{(20)}$. As a result, the study findings are not likely to be influenced by selection bias. This does not mean, however, that the study population of the present study is representative of the total population of CRC survivors. Indeed, the study included only a small proportion of stage IV CRC survivors, probably resulting from a survival effect, indicating that caution is warranted when generalising the findings to this particular subgroup of CRC survivors.

A limitation of the present study is that the data collection was mostly based on self-report, except for the clinical data that were collected through the ECR. This could have led to socially desirable answers, resulting in misreporting of specific health behaviours and related factors, which should be taken into consideration when interpreting our findings. For example, we observed that the dietary changes reported were predominantly in line with general recommendations for healthy eating, which might reflect an overestimation due to a social desirability bias. Additionally, 91\% of the survivors adhered to the Dutch physical activity guideline according to self-reported weekly levels of physical activity. Although comparable findings were previously observed in a similar cohort of Dutch CRC survivors ${ }^{(25)}$, physical activity levels were probably over-reported in the present study (according to the statistics of the National Public Health Compass in The Netherlands in 2012, 69\% of Dutch adults aged over 65 years adhered to the physical activity guideline).

Another limitation concerns the cross-sectional nature of our analyses. Even though part of the data was retrospective in nature (e.g. the clinical data from the ECR and items about changes since diagnosis) and our principal goal was not to find causal relationships, the predominantly nonlongitudinal nature of our analyses should be considered when interpreting the results, for instance with regard to 
self-reported dietary changes since diagnosis. Based on our cross-sectional data, we cannot know when the dietary changes occurred and if survivors attained to these changes. Presumably, however, the reported changes most probably represented permanent (rather than transient) dietary changes, perceived by the survivors as the most radical changes in their dietary habits after diagnosis, which were (still) being attained to at the time of the survey. Moreover, other lifestyle behaviours and related factors (i.e. physical activity, smoking, alcohol consumption and BMI) did not change from the first to the third survey of the ongoing longitudinal study (data not shown). This would suggest that if the survivors had changed their lifestyle, including dietary habits, these changes might predominantly have been made in the period early after diagnosis or treatment.

In conclusion, we have found that a substantial proportion of CRC survivors appears to alter their diet after diagnosis and use dietary supplements. These lifestyle habits appear to be adopted in part for different reasons and by survivors with partially distinct characteristics. Insights into the characteristics and motivations of CRC survivors who are receptive to selfprotective behavioural changes in lifestyle habits can provide leads for the design and tailoring of future interventions, and guide counselling strategies for promoting a healthy lifestyle.

\section{Supplementary material}

To view supplementary material for this article, please visit http://dx.doi.org/10.1017/S0007114515001798

\section{Acknowledgements}

The present study was supported by a grant from the Alpe d'HuZes Foundation within the research programme 'Leven met kanker' of the Dutch Cancer Society (grant no. UM-20125653). In addition, the present study was supported by a VENI grant (\#451-10-041) from the Netherlands Organization for Scientific Research awarded to F. M. M. J. B. (partly) and J. J. B.-P. are supported by a grant from Kankeronderzoekfonds Limburg as part of Health Foundation Limburg (grant no. 00005739).

The authors' contributions are as follows: M. J. B., S. B., R. M. W., F. J. v. D., F. M., E. K., M. P. W. and L. V. v. d. P.-F. were involved in the formulation of the research question(s) and study design; S. B., F. M. and L. V. v. d. P.-F. carried out the study; M. J. B., S. B. and R. M. W. performed the data analysis; M. J. B., S. B., R. M. W., F. J. v. D., F. M., J. J. B.-P., E. K., M. P. W. and L. V. v. d. P.-F. wrote the article.

There are no conflicts of interest.

\section{References}

1. Brenner H, Kloor M \& Pox CP (2014) Colorectal cancer. Lancet 383, 1490-1502.

2. Ferlay J, Soerjomataram DikshitR II, et al. (2014) Cancer incidence and mortality worldwide: sources, methods and major patterns in GLOBOCAN 2012. Int J Cancer 136, E359-E386.

3. Durko L \& Malecka-Panas E (2014) Lifestyle modifications and colorectal cancer. Curr Colorectal Cancer Rep 10, 45-54.
4. Vineis P \& Wild CP (2014) Global cancer patterns: causes and prevention. Lancet 383, 549-557.

5. Brenner H, Bouvier AM, Foschi R, et al. (2012) Progress in colorectal cancer survival in Europe from the late 1980s to the early 21st century: the EUROCARE study. Int J Cancer 131, 1649-1658.

6. Denlinger CS \& Barsevick AM (2009) The challenges of colorectal cancer survivorship. J Natl Compr Canc Netw 7, 883-893; quiz 894.

7. Thong MS, Mols F, Wang XS, et al. (2013) Quantifying fatigue in (long-term) colorectal cancer survivors: a study from the population-based patient reported outcomes following initial treatment and long term evaluation of survivorship registry. Eur J Cancer 49, 1957-1966.

8. Thong MS, Mols F, Lemmens VE, et al. (2011) Impact of chemotherapy on health status and symptom burden of colon cancer survivors: a population-based study. Eur $J$ Cancer 47, 1798-1807.

9. Thong MS, Mols F, Lemmens VE, et al. (2011) Impact of preoperative radiotherapy on general and disease-specific health status of rectal cancer survivors: a population-based study. Int J Radiat Oncol Biol Phys 81, e49-e58.

10. Demark-Wahnefried W \& Jones LW (2008) Promoting a healthy lifestyle among cancer survivors. Hematol Oncol Clin North Am 22, 319-342, viii.

11. Demark-Wahnefried W, Aziz NM, Rowland JH, et al. (2005) Riding the crest of the teachable moment: promoting long-term health after the diagnosis of cancer. J Clin Oncol 23, 5814-5830.

12. Ligibel J (2012) Lifestyle factors in cancer survivorship. J Clin Oncol 30, 3697-3704.

13. Anderson AS, Steele R \& Coyle J (2013) Lifestyle issues for colorectal cancer survivors - perceived needs, beliefs and opportunities. Support Care Cancer 21, 35-42.

14. Schmitz KH, Courneya KS, Matthews C, et al. (2010) American College of Sports Medicine roundtable on exercise guidelines for cancer survivors. Med Sci Sports Exerc 42, 1409-1426.

15. Rock CL, Doyle C, Demark-Wahnefried W, et al. (2012) Nutrition and physical activity guidelines for cancer survivors. CA Cancer J Clin 62, 243-274.

16. World Cancer Research Fund/American Institute for Cancer Research (2007) Food, Nutrition, Physical Activity, and the Prevention of Cancer: A Global Perspective. Washington, DC: AICR.

17. Buffart LM, Galvao DA, Brug J, et al. (2014) Evidence-based physical activity guidelines for cancer survivors: current guidelines, knowledge gaps and future research directions. Cancer Treat Rev 40, 327-340.

18. Maunsell E, Drolet M, Brisson J, et al. (2002) Dietary change after breast cancer: extent, predictors, and relation with psychological distress. J Clin Oncol 20, 1017-1025.

19. Stewart DE, Cheung AM, Duff S, et al. (2001) Attributions of cause and recurrence in long-term breast cancer survivors. Psychooncology 10, 179-183.

20. van de Poll-Franse LV, Horevoorts N, van Eenbergen M, et al. (2011) The Patient Reported Outcomes Following Initial treatment and Long term Evaluation of Survivorship registry: scope, rationale and design of an infrastructure for the study of physical and psychosocial outcomes in cancer survivorship cohorts. Eur J Cancer 47, 2188-2194.

21. van Duijn C \& Keij I (2002) Sociaal-economische status indicator op postcode niveau (Socioeconomic status indicator on zip code level). Maandstatistiek van de bevolking 50, 32-35.

22. Sangha O, Stucki G, Liang MH, et al. (2003) The SelfAdministered Comorbidity Questionnaire: a new method to 
assess comorbidity for clinical and health services research. Arthritis Rheum 49, 156-163.

23. Sprangers MA, te Velde A \& Aaronson NK (1999) The construction and testing of the EORTC colorectal cancer-specific quality of life questionnaire module (QLQ-CR38). European Organization for Research and Treatment of Cancer Study Group on Quality of Life. Eur J Cancer 35, 238-247.

24. Pols MA, Peeters PH, Ocke MC, et al. (1997) Estimation of reproducibility and relative validity of the questions included in the EPIC Physical Activity Questionnaire. Int J Epidemiol 26, Suppl. 1, S181-S189.

25. Buffart LM, Thong MS, Schep G, et al. (2012) Self-reported physical activity: its correlates and relationship with healthrelated quality of life in a large cohort of colorectal cancer survivors. PLOS ONE 7, e36164.

26. Soerjomataram I, Thong MS, Korfage IJ, et al. (2012) Excess weight among colorectal cancer survivors: target for intervention. J Gastroenterol 47, 999-1005.

27. Mullens AB, McCaul KD, Erickson SC, et al. (2004) Coping after cancer: risk perceptions, worry, and health behaviors among colorectal cancer survivors. Psychooncology 13, 367-376.

28. Satia JA, Campbell MK, Galanko JA, et al. (2004) Longitudinal changes in lifestyle behaviors and health status in colon cancer survivors. Cancer Epidemiol Biomarkers Prev 13, 1022-1031.

29. Stein KD, Kaw C, Crammer C, et al. (2009) The role of psychological functioning in the use of complementary and alternative methods among disease-free colorectal cancer survivors: a report from the American Cancer Society's studies of cancer survivors. Cancer 115, Suppl. 18, 4397-4408.

30. Van Loon K, Wigler D, Niedzwiecki D, et al. (2013) Comparison of dietary and lifestyle habits among stage III and metastatic colorectal cancer patients: findings from CALGB 89803 and CALGB 80405. Clin Colorectal Cancer 12, 95-102.

31. Robison LL \& Demark-Wahnefried W (2011) Cancer survivorship: focusing on future research opportunities. Cancer Epidemiol Biomarkers Prev 20, 1994-1995.

32. Richards M, Corner J \& Maher J (2011) The National Cancer Survivorship Initiative: new and emerging evidence on the ongoing needs of cancer survivors. $\mathrm{Br} J$ Cancer 105 , Suppl. 1, S1-S4.

33. James AS, Campbell MK, DeVellis B, et al. (2006) Health behavior correlates among colon cancer survivors: NC STRIDES baseline results. Am J Health Behav 30, 720-730.

34. Patterson RE, Neuhouser ML, Hedderson MM, et al. (2002) Types of alternative medicine used by patients with breast, colon, or prostate cancer: predictors, motives, and costs. J Altern Complement Med 8, 477-485.

35. Patterson RE, Neuhouser ML, Hedderson MM, et al. (2003) Changes in diet, physical activity, and supplement use among adults diagnosed with cancer. J Am Diet Assoc 103, $323-328$. 